animals. If, on the other hand, pure gamma globulin was used glomerulonephritis was the most prominent change in nearly all the animals.

The capricious localization is expressed clinically by the many forms of disease in man that seem to be hypersensitivity reactions of the kind $I$ have just described, such as polyarthritis acuta, endocarditis, pancarditis, periarteritis nodosa, arteritis temporalis, rheumatic pneumonia, transient infiltration of the lungs, glomerulonephritis, lupus erythematosus disseminatus, Liebman-Sachs disease, urticaria, angioneurotic oedema, migraine, etc. These diseases, however, may also occur in numerous combinations. It happens sometimes, however, that so many of these socalled diseases appear simultaneously in the same person that it is doubtful how the case should be designated.

Thus, whereas the anaphylactic type of hypersensitivity reaction, at least in its most advanced form, presents a characteristic pathologico-anatomical picture, this is by no means true of the bacterial hypersensitivity reaction, as it appears, for instance, in the tuberculin reaction, which resembles an ordinary inflammation.

With regard to contact dermatitis, the pathological anatomy is of no help whatever in deciding whether this is of anaphylactic or bacterial type. For long there has been controversy on whether the changes are primarily localized to the epidermal cells or whether they are secondary to changes in the capillaries of the cutis. Haxthausen has recently made experiments that support the latter assumption.

\section{Erythema Nodosum}

Finally, in order to show the importance of distinguishing between the various hypersensitivity reactions, I would like to say a few words about a disease which has traditionally interested Swedish research workers-erythema nodosum. In 1921 Ernberg coined the slogan that erythema nodosum is the body's own tuberculin reaction. This statement contains two important assertions-first, that erythema nodosum is always caused by tuberculosis ; secondly, that erythema nodosum is a hypersensitivity reaction of the bacterial type. The former declaration has been strongly opposed and successfully refuted. We know now that erythema nodosum also occurs in connexion with lymphogranuloma inguinale, streptococcal infections, etc. Little interest has, however, been taken in the latter assertion, though it has in general been accepted. But it might also be possible that erythema nodosum is a hypersensitivity reaction of anaphylactic type.

This view is not unreasonable. Already in 1929 Tillet and Frances showed that patients who are convalescent after pneumonia may show an immediate reaction to the carbohydrate component of the pneumococcus and a delayed reaction, similar to the tuberculin reaction, to the protein component. It is also known that an anaphylactic reaction can be produced by the carbohydrate component of the tubercle bacillus. Thus it is possible that in a tuberculous infection we have two parallel sensitizations-one to the protein component of the tubercle bacillus which results in bacterial hypersensitivity and is manifested in the tuberculin reaction, the second an anaphylactic reaction to the carbohydrate component of which erythema nodosum is an expression. That erythema nodosum often occurs when the tuberculin reaction becomes positive would then only mean that it takes approximately the same time for the two kinds of antibodies to form. This rule, however, is not quite without exceptions, for sometimes erythema nodosum occurs before the positive tuberculin reaction.
The clinical symptoms of swelling of the joints, urticaria, etc., which sometimes accompany erythema nodosum give additional support to this theory.

In my opinion the pathological changes in erythema nodosum also point in the same direction. For in the subcutaneous fatty tissue we find fibrinoid degeneration of the interstitial connective tissue, as has recently been shown by Wahlgren and Löfgren, and sometimes also vascular changes of the periarteritis nodosa type. I have myself had an opportunity of demonstrating this.

This question can naturally be solved only by testing patients suffering from erythema nodosum with partial antigens from tubercle bacilli and streptococci in order to find out whether they show any immediate reaction against these antigens. Strangely enough, investigations of that kind do not seem to have been made.

Finally, I should like to stress the importance of correlating our clinical, serological, and pathologico-anatomical knowledge of these problems better than in the past. The theory that the great number of clinical entities that I have mentioned here are only anaphylactic tissue reactions would then perhaps not be relegated to the world of fancy, as now quite often happens.

\section{THE STRUCTURE AND FUNCTIONS OF THE SYNOVIAL MEMBRANE*}

\section{BY}

D. V. DAVIES, M.A., M.B.

From the Anatomy Department, St. Thomas's Hospital Medical School

A thorough knowledge of the structure, nature, and functions of the synovial membrane is essential both for an understanding of the normal functioning of joints and for the interpretation and assessment of the changes which occur in disease. Despite the undoubted importance of this topic, knowledge of it is still to a great extent rudimentary. The literature is full of conflicting statements and is lacking in original observations, while textbooks on physiology in general tend to shun the problem. It is not intended to give here a comprehensive review of the subject, but to discuss some aspects in the light of my experience over the past twelve years. A few of the major problems remaining unsolved are raised in the hope that further investigation will be encouraged.

\section{Development}

The synovial membrane is the last of the joint tissues to be fully differentiated, and, as compared with the capsular structures and the skeletal elements, it is the least specialized, as shown by the frequency with which it is the source of heterotopic chondrification and ossification. The skeletal elements first take the form of mesodermal condensations (precartilage), which in turn are converted into cartilage and ultimately bone. Between the chondrifying skeletal elements is undifferentiated mesoderm-the so-called interzonal mesoderm or articular disk marking the site of the future joint and limited externally at an early stage by a cuff of condensed tissue delineating the capsular ligaments. Proximally and distally this capsular condensation blends with the more tardily differentiated perichondrium and later periosteum clothing the skeletal elements.

*Read at the combined meeting of the Sections of Orthopaedics and Rheumatology at the Annual Meeting of the British Medical Association, Harrogate, 1949. 
The chondrified skeletal elements expand and grow both by proliferation of their constituent cells with the accretion of matrix among them-the so-called interstitial method of growth-and by a new deposit of cartilage at their surfaces, the mesoderm here proliferating and transforming through a stage of precartilage to cartilage. At this stage the enveloping layer of precartilage is characterized by the density of its nuclei and its avascularity. The more peripherally placed mesoderm is a reticulum later destined to be the osteogenic layer of the periosteum. The fibrous layer of the periosteum later limits this externally and is from the first continuous with the capsular ligaments. At the ends of the skeletal elements and over the future articular surfaces the precartilage and cartilage are derived from the interzonal mesoderm. Between the articular surfaces this remains avascular, whilst the more peripheral portions between the capsule and the intra-articular parts of the skeleton are early and profusely vascularized (Haines, 1947). The synovial membrane is developed from this vascular mesoderm, and its rich capillary bed remains one of its most distinctive features. The central parts, and later the more peripheral of the interzonal mesoderm, cavitate to form the future synovial cavity of the joint (Fig. 1). This

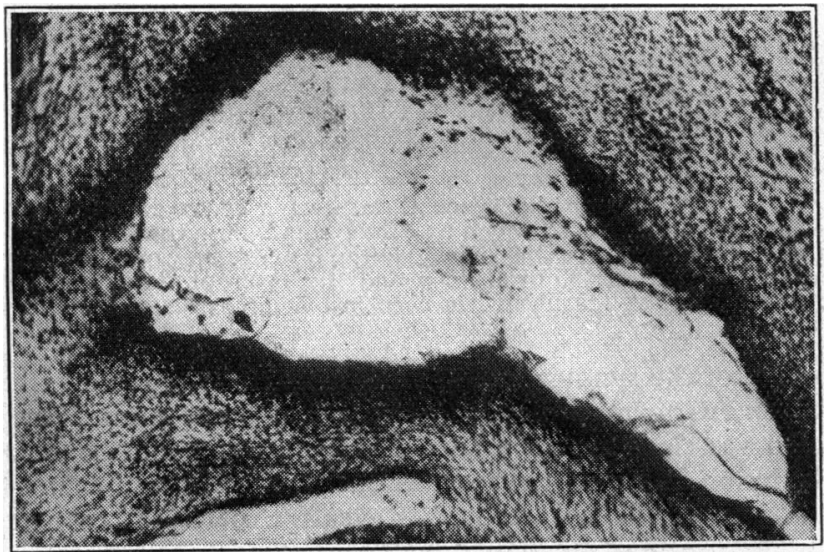

Fig 1-Section through the developing temporo-mandibular joint of a human embryo to show the process of cavitation to form the synovial cavity. ( $\times$ 120.)

is achieved by enlargement and confluence of the meshes of the reticulum by retraction and detachment of its constituent protoplasmic bridges, thus allowing adjacent spaces to unite and form a composite large space. Liquefaction of tissue plays a minor part in the process, but cannot be excluded. A few cells become free in the joint cavity and constitute the first cellular elements in the synovial fluid. Cavitation is often incomplete in these early stages and may continue even into post-natal life. Thus, of the original mesoderm of the interzone, that over the articular surface and intracapsular regions is converted into cartilage, while more peripherally the vascular layer continues as the synovial membrane lining the capsule and the intracapsular parts of the skeletal elements. The intra-articular ligaments are also developed from this mesoderm, and are delineated even before cavitation begins.

The major synovial folds of joints such as the infrapatellar pad in the knee are mapped out as cavitation proceeds, although we are ignorant of the time and method of deposition of the fat.

Villi are absent in the earliest-formed synovial membrane; they do not represent tags resulting from imperfections or irregularities of the process of cavitation. They arise by proliferations of the synovial membrane and not by delamination from its surface. A few villi are present at birth ; they continue to increase in number throughout life (Bennett, Waine, and Bauer, 1942).

Thus the significant features in the development of the synovial membrane are its slowness in attaining full differentiation, its early and pronounced vascularity, and the continuity of its parent tissue with chondrogenous tissue and later the osteogenic layer of the periosteum. The precocity of development and richness of the vascular bed denote its importance in the nutrition of the joint tissues, whilst its tardy differentiation and its genetic relation to cartilage and bone explain the frequency with which it is the site of heterotopic bone and cartilage formation. The marginal osteophytes of osteoarthritis develop in the region of transition and instability at the articular margin. Cartilaginous and bony loose bodies may arise in any portion, the latter either by ossification in cartilage or directly as i $\mathrm{t} \mathrm{r}$ a membranous ossification without the intervention of cartilage. Indeed, cartilaginous and bony nodules may arise together in the

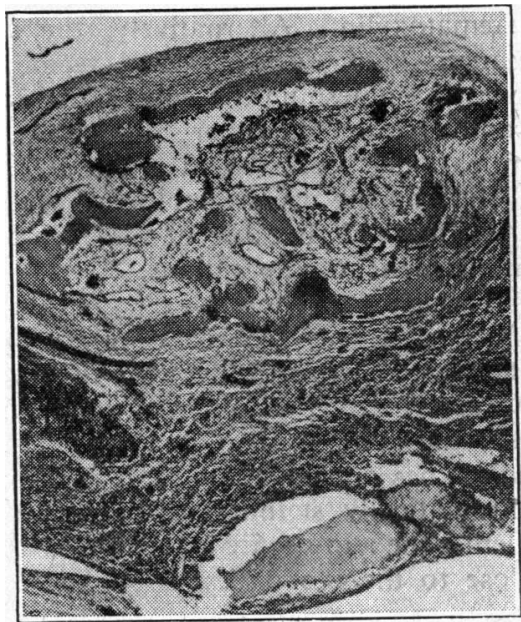

FIG. 2.-Section of the synovial membrane from a human hip-joint showing the formation of bony and cartilaginous loose bodies in close proximity to each other. $(\times 35$.) same synovial membrane and in close proximity (Fig. 2). When detached into the joint cavity as loose bodies these nodules remain enveloped in synovial cells, which may continue to proliferate cartilage, or more rarely bone, so that in time they may become very large.

\section{Components of the Synovial Membrane}

The synovial membrane as a whole varies considerably in its appearance from one part of the joint to another. Key's (1932) classification of the types as areolar, fibrous, and fatty adequately represents this range of variation. The fibrous type is in general thin and is placed directly on the ligaments of the joint, while from the physiological standpoint the loose areolar areas seem most active.

\section{The Synovial Cells}

Much discussion has centred on the nature of the synovial cells, their function, and their similarity or otherwise to the lining cells of serous cavities such as the pleural and peritoneal. They have been variously termed endothelial, mesothelial, and even epithelial. Some maintain that they differ little if at all from the underlying fibroblasts. Willis (1948) speaks of synovial fibroblasts at the surface of the membrane, merging without clear line of demarcation into collagen fibroblasts deeply, and at the articular margins showing transitions between typical synovial fibroblasts and cartilage cells. Key (1925) maintains that after synovectomy the new lining is re-formed by metaplasia of the underlying connective-tissue cells. These views are not wholly in accord with other observations. Unlike the lining of the serous cavities, these cells are irregular and ill-apposed; nevertheless in most histological preparations they differ from the underlying tissues by their depth of staining. With Mallory's connective-tissue stain they are characteristically coloured pink, whereas the 
underlying cells are blue. Like peritoneal cells, they prevent adhesions between apposed surfaces. Such a function could not be attributed to fibroblasts. To what extent adhesions occur after synovectomy it is difficult to tell. Like peritoneal cells, they react to irritation by becoming rounded and enlarged and by multiplying. Few synovial cells appear in either the normal or the pathological synovial fluid. Their characters here are adequately described by Collins (1936) and others. They and the other cellular elements in the fluid are most conveniently observed in films fixed wet for 20 to 30 minutes with Schaudin's or Bouin's fluid and then stained with Giemsa's stain or iron haematoxylin. Alternatively, the synovial fluid may be poured into Susa fixative, when the mucin is precipitated, enmeshing the cells. The coagulum thus obtained can be treated like other solid tissues, dehydrated, embedded in paraffin, sectioned at about $5 \mu$, and then stained on a slide by the usual histological methods. I have outlined these methods because of their simplicity and convenience as permanent preparations.

Though the synovial cells are almost certainly the main, if not the only, source of synovial mucin, they do not stain metachromatically with such stains as toluidine blue or cresyl violet as do many mucin-secreting cells. The essential component of the synovial mucin, the hyaluronic acid, does not possess this staining property. A few metachromatically staining cells can be seen in the synovial membrane ; these are always placed more deeply, and lie close to the blood vessels. They are mast cells (Davies, 1943).

In connexion with the nature of the synovial cells, the work of Vaubel (1933) merits special mention, and could be repeated and extended with advantage. According to that author synovial cells are distinguishable in tissue culture by their ability to liquefy the protein coagulum on which they grow-presumably secreting a proteolytic enzyme - and by their ability to secrete a mucin-like substance. Furthermore, their characteristic appearance in certain so-called synoviomata, and their ability to secrete a mucin here, point to them as the source of this substance and distinguish them from ordinary fibroblasts.

In addition the synovial cells have other important functions. They are active in the removal of particulate matter from the joint cavity and its transfer to the deeper tissues. Following haemorrhage into the joint cavity they are often loaded with haemosiderin. Routine examination for this pigment reveals its frequency, particularly in association with loose bodies in joints (Fig. 3). Large accumulations of haemosiderin frequently occur in the synovial cells also in villo-nodular synovitis, an inflammatory condition often erroneously termed xanthoma. Similarly, these cells prob-

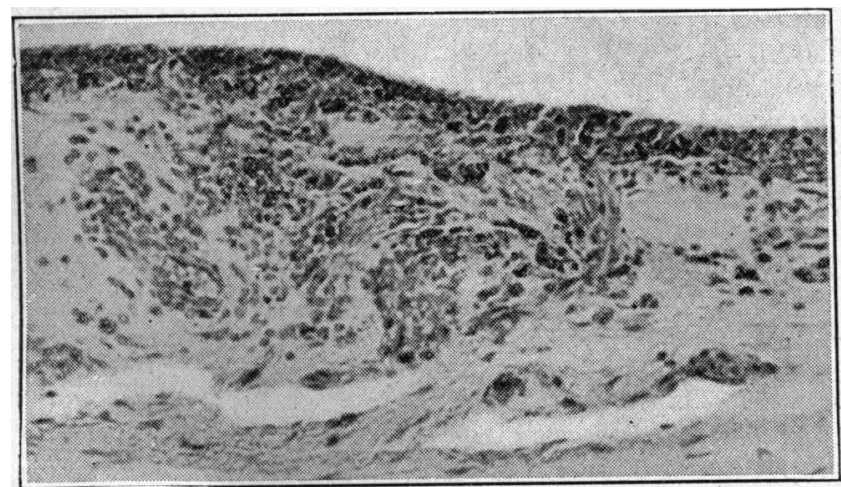

FIG. 3-Section of the synovial membrane of the knee-joint from a case with multiple loose bodies, showing the accumulation of pigment in the synovial cells and around the superficial blood vessels. (X 180.) ably participate in the removal of fibrin from the joint cavity in disease. Fibrinogen does not occur in normal. synovial fluid but is often seen in pathological conditions. Interesting in this respect are lesions found in the metatarsophalangeal joints of cattle three weeks after the intravenous injection of the vole bacillus vaccine (Young and Paterson, 1948). The animal is pyrexial, lame, and off its food; the joint becomes hot, swollen, and tender. At this stage the synovial membrane is swollen, oedematous, and hyperaemic, and the synovial cells are enlarged and increased in number ; the fluid in the joint is also increased and shows a high cellular content predominantly polymorphonuclear (Fig. 4). Fibrinous loose bodies are numerous and the

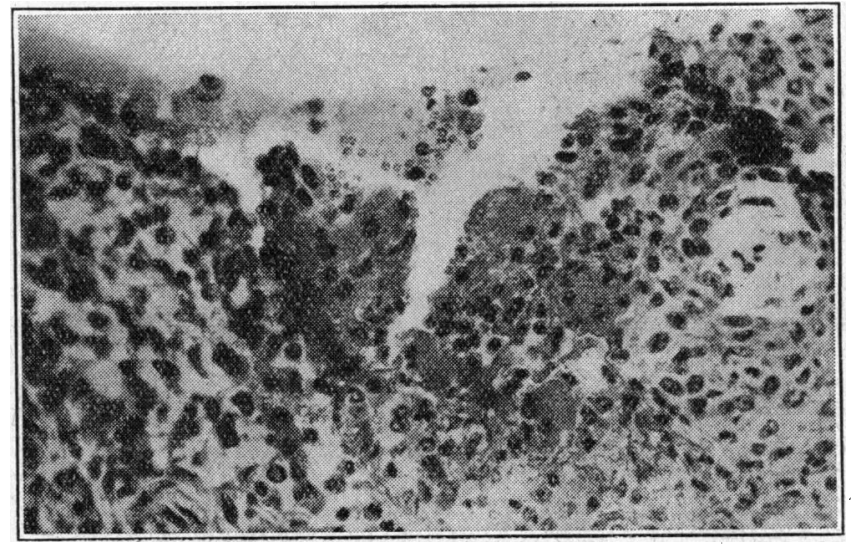

Fig. 4.-Section of the synovial membrane from a metatarsophalangeal joint of an ox which had received an intravenous injection of vole bacillus vaccine approximately three weeks previously. Note the reaction of the synovial cells and the layer of fibrin on the surface. Most of the fibrin has been lost in preparing the section. (X 300.)

surface of the synovial membrane is generally covered with a fibrinous membrane. The connective tissue around the blood vessels is degenerated and stains deeply eosinophilic. The lesion improves towards the end of a week, and examination after clinical recovery reveals a perfectly normal synovial membrane; the connective tissue is again normal, the synovial cells have returned to the usual size and arrangement, whilst the fibrin deposits have vanished, leaving no suggestion that they have become organized or incorporated into the membrane (Davies, 1948). The fibrin has presumably been lysed and absorbed. Vaubel (1933) notes the fibrinolytic properties of the synovial cells in tissue culture. This emphasizes the importance of the synovial cells in maintaining the joint fluid free not only of colloidal and particulate matter but also of extraneous protein.

\section{Villi; Fat Pads}

It would be appropriate at this stage to consider the villi of the synovial membrane. As a rule more highly developed in other animals than in man, they have been shown by Bennett, Waine, and Bauer (1942) to become more numerous with age. Their overgrowth in certain pathological conditions is truly astounding. They have been variously held to be the source of synovial fluid and concerned in the removal of particulate matter from the joint cavity. Frequently, however, they are avascular, and as regards the removal of particulate matter do not compare in activity with the areolar areas of the membrane. What, then, can their function be ? They increase the surface area of the synovial membrane, and possibly assist in maintaining the joint free of extraneous protein and allied substances. As yet, however, no close relation has been shown to exist between the capacity of the joint or surface, the volume of synovial fluid, the area of the articular surfaces, and the 
area of the synovial membrane. The data available are too scanty for any definite conclusion to be drawn.

Likewise, to the fat pads, almost universally present, have been ascribed a variety of functions-secretion of synovial fluid, filling of dead space, distribution of synovial fluid, and action as pad oilers. They are relatively inactive in regard to both absorption from the joint and the secretion of synovial fluid. There is little in the way of precise and authoritative information about this fat in the chronic wasting diseases or in purely arthritic lesions.

\section{Other Cellular Components}

Concerning the other cellular components of the synovial membrane, Kuhns and Weatherford (1936) have drawn attention to the reticulo-endothelial elements. Subcutaneous injections of trypan blue in animals lead to a considerable deposition of the dye in cells at or close to the synovial surface but excluding the fat pads. The storage of dye is not so marked as in sites, such as the liver, spleen, and bone marrow, known to be rich in reticulo-endothelial tissue, and it is increased by inflammation. Those authors suggest that these cells are of importance in the localization not only of known bacterial infections but also of bacterial toxins and metabolic products. The distribution of gold following chrysotherapy maintains the pattern described above: it is then present in the synovial membrane in smaller concentration than in liver and kidneys but greater than in muscle, and in greater concentration in arthritic joints than in normal synovial membrane (Davidson, 1948).

Though the collagen fibres of the membrane are of the utmost importance in the pathology of joint lesions, little that is certain can be said of their development and behaviour in normal tissue. The development and fate of the elastic fibres under varying conditions still require investigation. They protect the synovial folds from being nipped between the joint surfaces in the various movements of the joint.

Apart from its richness, already noted, the capillary bed of the synovial membrane is superficially placed, and

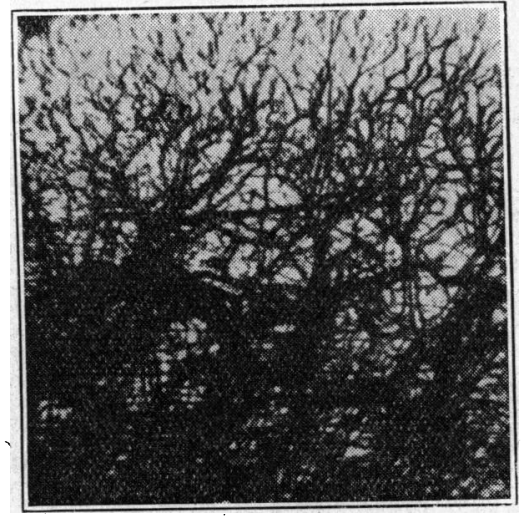

Fig. 5.-Human synovial membrane from the knee-joint showing the blood vessels of the circulus vasculosus. These nourish the peripheral parts of the articular cartilage and also the cartilage and later bone of the epiphysis. ( $\times 27$. around the articular margin forms the anastomosing fringe of capillary loops of the circulus vasculosus (Fig. 5). This is directly concerned in the nourishment of the peripheral portions of the avasc u l a r articular cartilage, the more central portions of which are nourished indirectly through the synovial fluid (Fisher, 1923). Apart from its mucin, this fluid is now believed to be a dialysate of the blood (Bauer, Ropes, and Waine, 1940). The capillary bed is also the chief site of absorption of solutions, and, to a smaller extent, colloidal particles from the joint-the less conspicuous lymphatic bed with its rectangular pattern and lacunae playing a more minor part (Adkins and Davies, 1940). As would be expected both from the structure of the synovial membrane and from its profuse vascularity, oedema of the looser areolar areas is a frequent feature in disease. Small haemorrhages and even thromboses are also often seen here, whilst the multiple terminal capillary loops help to determine the localization of infection.

\section{Innervation}

Finally the problem of the innervation of the synovial membrane constitutes one of the most controversial aspects of the whole topic. In this connexion the work of Gardner (1942, 1944, 1948), upon which I have drawn freely, needs special mention. Many conflicting statements appear in the literature: some describe nerve nets and even specialized nerve endings in the synovial membrane; others deny their existence. The nerves are few and always follow the blood vessels, close to which they terminate. No free nerve endings occur except in association with blood vessels and no complex end-organs are found (Gardner, 1948). My own more limited observations are in agreement with those of Gardner. This at once raises the question of the sensations arising in the membrane. In its looser and fatty areas it could hardly be expected to be concerned in the postural sensations. Is the membrane sensitive to pain, to touch, or to pressure ? I have once had the opportunity of testing this in a patient with a subacute infective lesion: touching the membrane produced no response, nor did pricking with a needle, nor pinching with forceps. Tugging on it, however, was sharply resented, though the pain was poorly localized. This pain may have been mediated through nerve endings situated in or close to the capsular ligaments. The mediation of pain sensations along fibres, presumably of the autonomic nervous system, situated on the blood vessels, is also a possibility. While, then, the synovial membrane is of the greatest importance from the point of view of nutrition of the joint, the part played by it in the symptomatology of disease is open to question. Engel (1941) has reported somewhat paradoxical results from division and stimulation of the sympathetic supply to perfused joints, the former apparently decreasing the permeability of the capillary bed here and the latter increasing it: this worker's interpretation is open to some doubt, and, in view of the paucity of nerve supply even to the vascular bed, his results may have been obtained by diversion of more blood to other regions following sympathectomy, or vice versa.

These, then, are a few of the problems which confront us. The field is a difficult one, but full of opportunities; the accumulation of data has so far been slow, and it is hoped that the near future will supply the solution to some of the basic problems involved.

\section{REFERENCES}

Adkins, E. W. O., and Davies, D. V. (1940). Quart. J. exp. Physiol., 30, 147 .

Bauer, W., Ropes, M. W., and Waine, H. (1940). Physiol. Rev., 20, 272.

Bennett, G. A., Waine, H., and Bauer, W. (1942). Changes in the Knee Joint at Various Ages. Commonwealth Fund, New York. Collins, D. H. (1936). J. Path. Bact., 42, 113.

Davidson, L. S. P. (1948). Textbook of the Rheumatic Diseases, p. 120. Ed. W. S. C. Copeman. Livingstone, Edinburgh.

Davies, D. V. (1943). J. Anat., Lond. 77, 160.

(1948). In Agricultural Research Council Report. (See Young and Paterson.)

Engel, D. (1941). J. Physiol., 99, 161.

Fisher, A. G. T. (1923). Lancet, 2, 541

Gardner, E. D. (1942). Anat. Rec., 83, 401. (1944). J. comp. Neurol., 80, 11. (1948). Stanford med. Bull., 6, 367

Haines, R. W. (1947). J. Anat., Lond., 81, 33.

Key, J. A. (1925). J. Bone Jt Surg., 7, 793. (1932). In E. V. Cowdry's Special Cytology, 2nd ed., 2, 1053. Hoeber, New York.

Kuhns, J. G., and Weatherford, H. L. (1936). Arch. Surg., 33, 68.

Vaubel, E. (1933). J. exp. Med., 58, 63.

Willis, R. A. (1948). The Pathology of Tumours. Butterworth,

London.
Young, J. A., and Paterson, J. S. (1948). "A A Progress Report on the Study of Vaccination of Cattle against Tuberculosis with the Vole Acid-fast Organism." Agricultural Research Council Report. 